\title{
La corrupción y estrategias anticorrupción: algunas cuestiones y estudio de casos de países en vías de desarrollo
}

\section{Alan Doig y Stephen Riley}

\section{Introducción}

Los modelos de corrupción varían de una sociedad a otra y a lo largo del tiempo. Así ocurre concretamente cuando analizamos el "Sur", el "Tercer Mundo" o los países en vías de desarrollo. Para entender la inmensa variedad de sus orígenes, formas y efectos en los países en vías de desarrollo, deberíamos examinar los roles tanto de los que tienen intereses internos en las sociedades en vías de desarrollo (como los políicicos, los grupos de hombres de negocios y los funcionarios públicos junior), como de los actores externos (incluidas las compañías multinacionales occidentales y las instituciones financieras internacionales). Además, las estrategias de reforma deberían tener en cuenta que los contextos económicos, legales y políicos difieren enormemente los unos de los otros. Para que las estrategias anticorrupción sean eficaces, deben elaborarse atendiendo al entorno social en el que la corrupción tiene lugar.

Como resultado de esta variedad de modelos de corrupción, se plantean problemas a la hora de evaluar la actual diversidad de la corrupción y de los esfuerzos anticorrupción; muchos son partidarios de una única estrategia universal para luchar contra la corrupción. La intención de este capítulo es valorar la corrupción y las estrategias anticorrupción en Botswana, Ecuador, Hong Kong y Tanzania, así como los intentos de reducir el fraude en las aduanas en Mali y Senegal, a la luz de tales soluciones universales. El capítulo comienza esbozando el marco actual del sistema de gobierno y la liberación económica y políica en la que se sitúan muchos intentos de reforma de los países en vías de desa- rrollo. Se debe examinar esta nueva agenda para la reforma en relación con el gran renacimiento del interés en reducir los ejemplos visibles de corrupción dentro del sector público a finales de los 90. ¿Por qué ha habido una "erupción de la corrupción» en los últimos años? Más adelante, el capítulo esboza los temas clave que se deben tener en cuenta a la hora de luchar contra la corrupción. Una importante sección del capítulo examina la diversidad de la corrupción y los esfuerzos anticorrupción puestos de manifiesto en los casos que se examinaron durante el Grupo de Trabajo del Centro de Desarrollo de la OCDE y el UNDP-PACT. La discusión final se centra en las principales opciones estratégicas que se requieren a la hora de diseñar una iniciativa anticorrupción eficaz a corto plazo y vuelve a analizar las soluciones universales propuestas.

\section{Un marco: un buen gobierno y una buena forma de gobierno}

El debate académico y el mantenido desde el prisma de las políticas públicas sobre la naturaleza de la corrupción - y la eficacia de las estrategias anticorrupción - se ha modificado nutablemente por los acontecimientos acaecidos en los países en vías de desarrollo y en el anterior Bloque Soviético a mediados y finales de los 80. Los argumentos para frenar la corrupción tienen ahora lugar en un nuevo contexto: estamos en una era de reforma polííca y adminis- 
trativa, de ajuste estructural y lo que Samuel H un TIN GTON ha denominado la Tercera 0 la de la democratización en los países en vías dedesarrollo y en transición. Por tanto, a finales delos90, para alcanzar el objetivo de reducir la corrupción, se deben tener en cuenta estos imperativos de política internacional.

Sin embargo, la erradicación de la pobreza, el desarrollo de la participación y el apoyo a los derechos humanos han sido objetivos que han existido durante mucho tiempo (sobreel papel, aunque no sobre la práctica) en los países occidentales y en los organismos internacionales que éstos financian. Ya sea por el fin de la guerra fría y, por tanto, porque ya no es necesario apoyar liderazgos políticos en los países no alineados o en vías de desarrollo, ya sea porque se ha debilitado el énfasis que se hacía sobre la dimensión y roles del Estado, tras el fin de los planteamientos reaganianos 0 thatcheristas, estos objetivos han pasado a ocupar el primer lugar de la agenda, insistiendo claramente en que la financiación se dirigirá ahora a conseguirlos. Por ejemplo, el InformeFinal del Grupo deTrabajo Ad H oc sobre el D esarrollo de la Participación y el Buen Gobierno del Comité de Ayuda al D esarrollo de la OCDE [1997] ha subrayado como los temas claves: la reducción de la pobreza, la promoción de la igualdad de géneros, el establecimiento de los estándares básicos de la educación y de la salud e invertir la marcha de la degradación medioambiental. El informe de la OCDE, además de los de otros organismos y gobiernos, sugiere que tales temas se desarrollan mejor a través de sociedades más participativas, transparentes y responsables, haciendo hincapié en una reforma sostenida, que incluya la promoción del desarrollo y de la liberalización económicos, la mejora de las expectativas sociales, sanitarias y educativas de la población y la dotación de un marco político y legal responsable y comprometido.

Se espera que este marco - generalmente denominado «buen gobierno»- contenga algunos componentes clave: legitimidad política del Estado mediante elecciones democráticas y la consiguiente transferencia de poder, y una oposición política eficaz y un gobierno representativo; responsabilidad mediante la transparencia y suministro de información; separación de poderes; auditoría eficaz, tanto interna como externa; medios eficaces para luchar contra la corrupción y el nepotismo; un funcionariado competente, en el sentido de funcionarios públicos capacitadosy formados; políticas realistas y un bajo gasto en defensa; derechos humanos, tales como libertad de religión y movimiento; sistemas de justicia penal imparciales y accesibles; y la ausencia de un poder arbitrario del gobierno.

El buen gobierno también se considera una condición esencial en el camino hacia el objetivo más amplio de un buen sistema de gobierno. D escrito como el «uso de la autoridad política y el ejercicio del control sobrela sociedad y la gestión de sus recursos para el desarrollo social y económico», un buen sistema de gobierno abarca la «esencia del funcionamiento de los mecanismos estructurales e institucionales del Estado, los procesos de toma de decisiones, la formulación de las políticas, la capacidad deimplemen- tación, los flujos de información, la efectividad del liderazgo y la natural eza de la relación entre los go bernantes y los gobernados» (Serageldin y Lan deLL-M ILLS, 1991). Un buen sistema de gobierno, por tanto, no sólo tienequever con la organización y la actividad del gobierno, sino también con los fines que se le encomiendan de lograr ciertos niveles de desarrollo económico, humano einstitucional que «beneficien a la población en su conjunto» y promuevan «la alfabetización, la educación y las oportunidades deempleo», lo quea su vez aumenta la capacidad dela población para demandar y participar eficazmente en el buen gobierno (Banco M undial, 1991).

En términos generales, el buen gobierno es una precondición esencial de todo buen sistema de gobierno. El buen gobierno tiene como finalidad al canzar un marco gubernamental aceptado por un público tan participativo como legítimo; sensible a las necesidades de la población y comprometido con la mejora de su bienestar; con capacidad para imponer la ley y el orden y prestar servicios públicos; que establezca un entorno en el que se desarrollen políticas públicas eficaces; y que sea generoso en su conducta. Tal sistema de gobierno también querría evitar cualquier compromiso 0 implicación directa en otras áreas, concretamente en la producción económica, para concentrar sus esfuerzos y capacidades en las prioridades de la sociedad. U na vez que está en marcha la reforma política y se han desmantelado las economías controladas centralmente, este argumento sugiere que los principios liberales de mercado pueden empezar a promover el desarrollo económico que, a su vez, debería promover la participación en los procesos políticos. Los objetivos económicos y políticos a largo plazo se complementarían, por tanto, unos a otros para trabajar en la consecución de «una población instruida, con cultura política y voluntad de actuar, y ello unido a una economía industrial moderna, una sociedad homogénea y un conjunto de valores políticos democráticos muy arraigados» (RILEY; 1992, p. 18).

Tales condiciones deberían generar internamente una basefiscal, estable y sostenibley una clase económica importante que estuviera fuera del sistema político, cuyaimplicación o participación en losprocesos políticosy administrativos fuera menos por su propio beneficio que porque creyesen en la promoción y en los roles del Estado relativos a regular y reportar un beneficio a la sociedad, cuya confianza en, y la aceptación de, la legitimidad de la neutralidad y sensibilidad del Estado, se vería confirmada por los esfuerzos que esta clase económica habría realizado en nombre de la población en su conjunto, lo quea su vez reforzaríala estabilidad del Estado y la viabilidad financiera (T HEOBALD, 1993).

Por tanto, la nueva agen da política internacional de finales de los 90 recoge al gunos presupuestos sobre la corrupción y los medios que son eficaces para reducirla. La corrupción se define, evidentemente, como corrupción de los empleados públicos, del sector público o corrupción institucional. La discusión sobre la reforma dela corrupción tienelugar casi siempreen el contexto de un marco de sistema de gobierno e incluye expectativas optimis- 
tas de liberalización, tanto económica como política. También se da por sentado que la corrupción en el sector público se reducirá si se reduce el tamaño del Estado. También se minimizará la corrupción mediante la liberalización económica general y la liberalización política, es decir, con cambios que lleven hacia una política liberal, plural, lo que supone una prensa más libre, una política de partidos competitivos y el resurgimiento o creación de otras instituciones independientes, que reduzcan la corrupción al hacerla más vulnerable a la exposición pública. De esta forma, la corrupción tendrá consecuencias políticas potencialmente perjudiciales.

\section{Corrupción y cambio}

Los gobiernos y organismos están reconociendo cada vez más la "transcendencia estratégica que tiene el buen gobierno en el proceso de desarrollon y que el buen gobierno requiere de los "más altos estándares de integridad, franqueza y transparencia", así como sistemas de justicia penal sólidos y prestar atención a las "nuevas formas y dimensiones" de la corrupción y su efecto generalizado sobre la actuación del gobierno, sobre el uso de los recursos públicos, sobre la moral general en los servicios públicos y sobre la legitimidad del Estado y el Derecho (Naciones Unidas, 1989). Como condición previa para llevar a cabo una reforma sostenida, se ha enfatizado la necesidad de cambiar el tratamiento que se ha venido haciendo de la corrupción, mientras que el hecho de que sea evidente hacerlo así se ha considerado un componente clave del compromiso de alcanzar el buen gobierno.

La reacción ante y la preocupación por controlar la corrupción es, en parte, producto de los acontecimientos: la «erupción de la corrupción». En los 90 ha habido muchos ejemplos de corrupción dentro del sector público a los que se les ha dado mucha publicidad en sociedades muy diferentes: Italia, Corea del Sur, el Reino Unido, Kenia, Alemania y Sudáfrica, además de los casos examinados en este capítulo, han sufrido escándalos de corrupción importantes (IDS, 1996; HAYWARD, 1997). Sierra Leona y el Zaire de Mobutu (ahora la República Democrática del Congo) son ejemplos muy ilustrativos de los efectos enormemente perjudiciales de la corrupción sistemática ampliamente extendida y sus efectos en términos de cambio político (ASKIN y COLLINS, 1993; KPUNDEH, 1995). También hay una preocupación creciente por la corrupción, el fraude y el crimen organizado en la Europa del Este y la anterior Unión Soviética (REED, 1994; TranG, 1994; KaUfMANN, 1997).

La corrupción ya no parece ser sólo una enfermedad temporal de las sociedades en proceso de modernización, que curará la alfabetización, el desarrollo y una ética pública correcta. Los efectos sociales, políticos y económicos cada vez más debilitadores producidos tanto por la corrupción a pequeña y gran escala se pueden apreciar en cualquier parte. Como resultado, se ha hecho evidente la necesidad de hacer ahora explícito el compromiso por lograr un buen gobierno y, por tanto, un buen sistema de gobierno, que se puede haber presupuesto como implícito, tanto a través de programas de reforma detallados (como ha publicado el DAC de la OCDE [1997]), como por la amenaza de restringir la financiación de aquellos países que no logran actuar de forma eficaz para reducir la corrupción (como indicó el Banco Mundial en septiembre de 1997). Tales organismos también han proyectado el marco-macro y los temas-macro para, y las consecuencias de, comprometerse en conseguir un buen gobierno y el tema de abordar la corrupción ha llegado a ser un componente clave de la revitalización de la capacidad institucional del Estado (Banco Mundial, 1997), para asegurar la confianza de la sociedad en su liderazgo (Programa Internacional de Control de Drogas de $\mathrm{Na}$ ciones Unidas, 1997) y para proteger el tejido de la vida pública (PDNU, 1997). El número de organismos públicos y asociaciones profesionales que han realizado ya declaraciones o llevado a cabo políticas públicas sobre la corrupción y el fraude en el sector público es sustancial. Pero la preocupación más inmediata, tanto de los organismos como de los países implicados, es cómo abordar la corrupción en la práctica: qué estrategias de reforma a corto y largo plazo emplear y qué perspectivas sobre los modelos y variedades de corrupción adoptar para ofrecer un marco apropiado.

\section{Cuestiones al abordar la corrupción}

Se deben tratar algunas cuestiones para comprender la corrupción. Por ejemplo, ¿cuál es el impacto de las organizaciones 0 actividades externas? ¿Presentan las economías en vías de desarrollo situaciones de corrupción diferentes que varían en la forma, localización e intensidad de unos países a otros? Tales temas tienen que ver con que si la corrupción debería tratarse de la misma forma en todos los contextos políticos y económicos o si es incluso pertinente intentar desarrollar una estrategia universal para luchar contra la corrupción.

Es comparativamente complejo y metodológicamente delicado tratar de identificar causas o modelos genéricos de corrupción dentro de una escala temporal de desarrollo única o determinada, y dentro de las actividades del Estado o sin hacer referencia a temas colaterales. Aunque la corrupción en sí misma es un fenómeno antiguo para la que se han propuesto numerosos remedios (NoONAN, 1984; SEN, 1997), los estudios académicos enfocados desde el prisma de las políticas públicas han prestado una atención cada vez mayor a la definición, orígenes, naturaleza y costes de la corrupción en los países en vías de desarrollo desde los 60. A menudo, la 
corrupción ha sido un tema central de investigación por derecho propio, porque implica el socavamiento de los cargos públicosal utilizarseen ben eficio privado, pero también porqueestáintrincadamente vinculado con otras formas de anarquía y mala administración, incluidos el fraudey el crimen organizado. Su predominio y longevidad son motivo de preocupación por sus consecuencias públicas y sociales perjudiciales: mina las capacidades de desarrollo de los Estados, distorsiona las prioridades y es, con frecuencia, una forma de redistribución del dinero de aquellosque están en la pobreza a losempleados públicosque se están enriqueciendo (aunquehay al gunosejemplos en dondelos empleadospúblicos debajo nivel y a menudo mal pagados han respondido ante la imposibilidad del Estado de pagarles sus sal arios oficiales durante largos pe ríodos de tiempo vendiendo sus recursos - por ejemplo, la venta de drogas por parte de los empleados de los servicios de salud en África - para generar una fuente de ingresos equivalente). La corrupción también varía indefinidamente en cuanto a su naturaleza según los regímenes, institucionesy grupos en las distintas economías en vías de desarrollo y, a menudo, está sujeta a enfoques diferentes, dependiendo desu trascendencia política o de su impacto en la sociedad; por ejemplo, la «gran» corrupción o la corrupción de alto nivel versusla corrupción de bajo nivel; la corrupción administrativa y legislativa; o la corrupción en los distintos servicios públicos.

El interés académico y la preocupación en la elaboración de las políticas públicas respecto de los costes de la corrupción en los países en transición y en vías de desarrollo también han variado considerablemente desde los 60: los estudios generales de carácter teórico en los 60 y los 80 han dado paso a estudios sistémicos y trabajos empíricos más detallados, por ejemplo, en relación con la liberalización económica en la República Popular China. Las modas académicas se han hecho eco de las preocupaciones en la elaboración de las políticas públicas y de los «pánicos morales» sobre la corrupción. El actual aumento sustancial del interés académico y de las políticas públicas en los costes (perjudiciales) de la corrupción a mediados de los 90 es un producto de la preocupación cada vez mayor porque se percibe un crecimiento de la corrupción de cierta entidad en todo el mundo. D esde los 60, los debates académicos han reflejado las prioridades dentro de las políticas públicas deal gún benefactor y algún gobierno: con la modernización y el crecimiento infraestructural en los60; con la equidad y la universalización de los derechos a comienzos de los 80; y con el ajuste estructural, un buen (o mal) sistema de gobierno, la liberalización económica y política y la desregulación a finales de los 80 y 90 (CHARLICK, 1993; D OIG, 1995; H ARSCH, 1993; IDS, 1996; RILEY, 1993).

La corrupción incidental es un rasgo vital en casi todas las sociedades, pero puede ser sistemática en muchas instituciones públicas en los países en vías de desarrollo, si no sistémica en la sociedad en su conjunto (RILEY, 1983) y puedetambién ser endémica y planeada. En muchospaíses, unafuenteclave decorrupción pue deser (la clase política) el liderazgo político que sesirvea sí mismo y un marco estatal amplio, ineficiente y políticamente influenciado y mal dirigido, dentro del cual losinteresesprivadosindividuales y de grupo tienen prioridad sobreel bien colectivo. Los empleados públicos tienen un poder discrecional considerable para acumular riqueza privada a través de la explotación de sus posiciones monopolísticas, mal eirregularmente pagadas, a menudo en connivencia con los políticos y hombres de negocios autóctonos o extranjeros. La corrupción, por tanto, tienelugar frecuentemente en las sociedades donde los empleados públicos tienen un considerable poder discrecional, rinden cuentas de forma limitada y hay poca transparencia en las operaciones del gobierno; en tales sociedades, lasinstituciones dela sociedad civil y el sector privado independiente a menudo son débiles o están poco desarrollados. El establecimiento de la corrupción sobre una base sistemática o sistémica puede ser una consecuencia de la perpetuación de las desigualdades existentesy Estados débiles («blandos» 0 «huecos»), dondepuede desarrollarse muy bien «la desorganización, la ausencia de relaciones estables entre grupos y la ausencia de model os reconocidos de autoridad» (H UN TINGTON, 1968, p. 710; AN D RESKI, 1970), pero su permanencia puededescansar en una dinámica queseautosoporta, porque se sustituye o adapta según las circunstancias y el contexto y, por tanto, su fallecimiento no se «asociaría necesariamente con la modernización política. Tampoco la corrupción está condenada a la destrucción a medida que un sistema político madura. La corrupción altera su natural eza en respuesta a los factores socio-económicos, culturales y políticos cambiantes. Puesto que estosfactores afectan a la corrupción, aśl la corrupción también les afecta a ellos) (WERNER, 1983, p. 638). Al mismo tiempo, mientras que puede ser difícil comprender «que la administración pública puedeincluso aspirar a, y no digamos, mantener nivelesaceptables dehonradez y eficienciafrenteaunosantecedentes decastigo del endeudamiento, inflación gal opante, desempleo crónico, estándares de vida sombríos y las serias tensiones cívicas que tal es condiciones producen inevitablemente... , afirmar quela corrupción es simplemente una consecuencia del subdesarrollo implica correr el riesgo de aceptar un evolucionismo tosco que prevé una disminución proporcionada de la cantidad de abusos con cada mejora porcentual en el PIB»(ThEOBAL, 1990, p. 164).

\section{Entendiendo la diversidad: estudio de casos de páses en vías de desarrollo}

Se acepta con carácter general que la corrupción - ya sea incidental, sistemática o sistémica y ya sea endémica o planeada- 
tiene unos efectos políticos, sociales y económicos profundamente perjudiciales, aunque sus consecuencias políticas varían. $\mathrm{Ha}$ contribuido a la inestabilidad política, a la intervención militar y al cambio de régimen y ha socavado las instituciones gubernamentales, tales como las aduanas, la imposición fiscal y otros departamentos generadores de ingresos y la prestación de servicios (WADE, 1985), a menudo como consecuencia del funcionamiento de un orden político neo-patrimonial, como en el África Subsahariana (SANDBROOK, 1985). Mientras que las causas y las consecuencias de la corrupción son a veces difíciles de desenmarañar de los temas más novedosos y los efectos más amplios del fraude a gran escala, los movimientos de capital y el blanqueo de dinero (HAMPTON, 1996) y el crimen o los negocios organizados y el robo internacional (LEVI, 1987), se pone énfasis actualmente en los efectos distorsionadores de la corrupción (IRIS, 1996; GOUDIE y STASAVAGE, 1997; ROSE-ACKERMAN, 1978; SHLEIFER y VISHNY, 1993). Existe cada vez un mayor consenso internacional en el desarrollo de un discurso sobre el perjuicio que la corrupción puede causar a los pobres, al crecimiento económico y a la integridad pública (PDNU; 1997; Banco Mundial, 1997). Para los países en vías de desarrollo, este consenso sugiere que la democratización, los "recortes de plantilla" y la desregulación del sector público no son sólo objerivos deseables en sí mismos, sino que también son un medio útil para reducir la corrupción generalizada. El argumento general de que una forma importante de reducir la corrupción en el sector público es proceder a un ajuste estructural sostenido, mientras que también se liberaliza políticamente y se siguen realizando mejoras en el sistema de gobierno, sin embargo, precisa de una valoración dentro del contexto de las experiencias de corrupción de los países.

En el Grupo de Trabajo del Centro de Desarrollo de PDNUPACT y de la OCDE sobre "La corrupción y las iniciativas de mejora de la integridad en el contexto de economías en desarrollo", en París en 1997, algunos documentos ofrecieron algunos aspectos de modelos de corrupción, sus causas y respuestas. Los casos estudiados permiten identificar modelos de corrupción distintos, planteamientos específicos y posibles soluciones genéricas. También se precisa que la evaluación de las experiencias de los esfuerzos anticorrupción en Botswana, Ecuador, Hong Kong y Tanzania y los intentos de reducir el fraude aduanero en Mali y Senegal se deben situar en este nuevo contexto de política internacional.

La corrupción difiere considerablemente en los países en vías de desarrollo. A causa de esta diversidad, la corrupción es difícil de minimizar o controlar. Por tanto, es importante examinar los aspectos concretos de la corrupción y de los esfuerzos anticorrupción, incluidas las cuestiones relativas a la cultura, organización y la eficacia de las campañas, al tiempo que también se estudian las estrategias más universalistas que se han desarrollado en los últimos tiempos. A continuación, se recogen ejemplos de estrategias específicas.

\section{Botswana}

Durante muchos años, Botswana, como la anterior Colonia de Hong Kong de la Corona Británica, ha sido considerado como un ejemplo de país con niveles relativamente bajos y manejables de corrupción en un continente no caracterizado por niveles generalmente altos de integridad pública. Por la razón que sea, la élite política de Botswana parecía ser capaz de gestionar el problema de la corrupción, incluso cuando presidía un país con tasas envidiablemente altas de crecimiento económico (comparables a las tasas de crecimiento de NICs de Asia Oriental), un sistema multipartidista y un buen sistema de gobierno. En los 90 esta reputación se ha empañado por la aparición de una serie de escándalos de corrupción. Kwame FrIMPONG (FRIMPONG, 1997) sostiene que la corrupción llegó a ser un problema en los 90 como resultado de la «complacencia política" y de una suposición optimista de que el problema de la corrupción se había gestionado eficazmente. FRIMPONG identifica una serie de escándalos que rodean la compra por parte del gobierno de libros de texto para el colegio (que suponía una pérdida de 15 millones de dólares americanos), distribución de tierras y gestión de alojamiento. Los escándalos dieron lugar a tres Comisiones Presidenciales de Investigación en 1991 y 1992, que revelaron que los que estaban implicados se encontraban entre los mejor pagados del país. Estos ejemplos de gente relativamente rica que se hacía más rica a través de la corrupción son contrarios al punto de vista de que gran parte de la corrupción africana es un delito perpetrado por los peor pagados. Un partido gobernante muy mayoritario en Botswana había minado las garantías democráticas contra la corrupción, además de las tradicionales formas de exigencia de responsabilidades. Más tarde, el gobierno estableció un Consejo para la Corrupción y los Delitos Económicos (Directorate of Corruption and Economic Crime o DCEC) en 1994, que ha informado anualmente al Parlamento. Estos desarrollos reflejan un compromiso político para acometer tal cuestión, poniendo de manifiesto el compromiso adquirido con el número de casos investigados por el DCED, el número de casos que se han llevado ante los tribunales y el número de condenas. El Informe DCEC de 1996 señaló que "la anterior hipótesis de que la corrupción existía en muchas esferas y a todos los niveles, tanto en el Gobierno como en el Gobierno Local ... ha resultado ser correctan. Como la estrategia anticorrupción de Hong Kong, el DCEC de Botswana ha desarrollado un ataque tridimensional, centrado en la investigación y acusación, educación y prevención pública. FrIMPONG concluía su argumento sugiriendo que cualquier lucha eficaz contra la corrupción en los países en vías de desarrollo requiere de la colaboración internacional, por el impacto de la MNC's, del comercio y de otras interdependencias económicas.

\section{Ecuador}

El Dr. Ramiro Larrea-Santos (LARREA-SANTOS, 1997) dio un discurso en su rol de Presidente de la Comisión Anticorrupción 
de Ecuador. La Comisión Anticorrupción se creó en marzo de 1997 con la intención de canalizar las actividades de la sociedad civil en el descubrimiento, investigación y vigilancia de la corrupción. Los resultados del trabajo de la Comisión se entregarían a los poderes legislativo y judicial del Estado ecuatoriano. Las operaciones de la Comisión se extendieron hasta agosto de 1998 y recientemente ha desarrollado un anteproyecto de ley para institucionarse a sí misma.

Los efectos perniciosos de la corrupción en Ecuador han amenazado la propia estabilidad de su democracia. La corrupción se vinculaba a al gunos dramas políticos recientes del Estado, incluido el levantamiento del pueblo ecuatoriano contra el régimen anterior de Abdala Bucaram en febrero de 1997. M uchas actividades del Estado en el antiguo régimen se vieron afectadas por la corrupción: éstas incluían los servicios fiscales y de aduanas, la construcción de obras públicas y otros contratos públicos. Esto contribuía a una prestación de servicios completamente inade cuada y a la politización de las finanzas públicas y de la justicia. Los contratos públicos incluían sobornos de entre el $10 \%$ y el $30 \%$, mientras que los apagones de electricidad de hasta ocho horas al día eran en parte producto de la corrupción. La evasión de las obligaciones aduaneras era masiva, estimada en 210 sucres bolivianos en seis meses. El Dr. Larrea-Santos listó catorce «causas principales» de la corrupción y los once «efectos más visibles» dela corrupción en Ecuador. Las causas principales eran el colapso de los valores éticos, el analfabetismo y un Estado excesivamente centralizado, no transparentey politizado. Los efectos más visibles eran la violencia y un sentido de renuncia ante los efectos de la corrupción, los servicios públicos deficientes y un incremento de la polarización y exclusión social. Un gran número de iniciativas anticorrupción en las esferas legales, educacionales y sociales estaban en camino en ese momento, incluida la creación denuevos mecanismoslegales anticorrupción, actividades deeducación pública y la investigación y acusación de los empleados públicos del régimen de Bucaram. La participación popular y la conciencia cívica en todas las organizaciones de la sociedad civil fueron elementosimportantes en la estrategia anticorrupción, pero mientras que se reconocían como valiosos los temas sociales y educacionales (como la reducción del analfabetismo), a menudo tenían un impacto a largo plazo. Lo que era importante era la identificación de los remedios eficaces y a corto plazo.

El caso de Ecuador era también un recordatorio oportuno de que la corrupción podría haber tenido efectos políticos dramáticos: había contribuido a la explosión política en Ecuador a comienzos de 1997. Era discutible si había una relación directa entre la corrupción y la violencia; se ha sugerido en todas partes (por ejemplo, Samuel Huntington, en los 60) que la corrupción y la violencia eran alternativas en los países en vías de desarrollo. Por tanto, la corrupción no siempre conducía a la violencia, aunque a menudo incrementaba el cinismo y hacía disminuir la participación popular. Tanto la pequeña como la gran corrup- ción política eran, desde el punto de vista político, profundamente alienantes y podían tener efectos intensos a corto plazo, así como efectos perjudiciales a largo plazo.

\section{Hong Kong}

Bertrand de Speville (De SpeviLLe, 1997) estudió la guerra de H ong Kong contra la corrupción, desde el punto de vista de su anterior posición como Comisionado de la Comisión Inde pendienteC ontrala Corrupción (Independent Commission Against Corruption o ICAC). La ICAC es un órgano estatutario establecido en 1974 cuyo solo propósito es luchar contra la corrupción y que había desarrollado una estrategia de ataque de tres dimensiones. H ong Kong destacaba como ejemplo de sociedad en donde la incidencia de la corrupción había disminuido dramáticamente a lo largo del tiempo. La esencia de la contribución de DE SPEVILLE se centraba en una valoración de la traslación y adaptabilidad del «modelo de H ong Kong», los costes de la ICAC, el carácter preciso del modelo en sí mismo, el futuro de la corrupción en H ong Kong ahora que había dejado de ser una Colonia de la Corona Británica y dónde había interrogantes sobre el modelo de desarrollo asiático del N IC.

La corrupción había sido una vez un serio problema en Hong Kong: estaba ampliamente extendida, profundamente arraigada, bien organizada y tolerada. Afectaba a cada parte de los servicios públicos de la Colonia de la Corona. Las causas de la corrupción se relacionaban con el rápido crecimiento económico y demográfico, la población inmigrante llegada recientemente a $\mathrm{H}$ ong Kong (principalmente desde China, donde la corrupción era endémica) y el deseo de la administración de regular y controlar la economía. La corrupción también era un problema especialmente difícil en la fuerza policial. El desasosiego público respecto de la corrupción y la desaparición de un empleado público senior de la policía europea que estaba siendo investigado por cargos de corrupción, precipitaron la acción.

La estrategia anticorrupción abarcaba la investigación, prevención, educación y reclutamiento de apoyo. Un elemento clave de la estrategia era la ICAC, que operaba tanto en el sector público como en el privado y trataba de coordinar las distintas partes de la estrategia. Reclutó principalmente investigadores de la policía al principio, pero también empleó a funcionarios públicos, ingenieros, contables y expertos en medios de comunicación para las relaciones con la comunidad. La ICAC organizó estudios de prevención de la corrupción para los organismos públicos y compañías del sector privado. También dirigió campañas en los medios de comunicación y campañas de educación pública contra la corrupción. La naturaleza de las investigaciones cambió a lo largo del tiempo, a medida que se prestaba más atención a la corrupción en el sector privado como respuesta a las quejas públicas. En los 90, hubo procesamientos de miembros destacados del ámbito 
empresarial, incluido el Presidente de la Bolsa. Los efectos a largo plazo de la estrategia eran el incremento de la confianza de la comunidad y el apoyo al ICAC, un servicio público y empresarial más transparente y un mayor entendimiento de la corrupción y de por qué es perjudicial. En términos de futuro, DE SPEVILLE sostenía que Hong Kong no volvería de hurtadillas a la corrupción, dada su experiencia en luchar contra la corrupción, su status jurídico independiente y los compromisos tanto del pueblo de Hong Kong, como del gobierno chino.

Resulta problemático indicar hasta qué punto es trasladable el "modelo de Hong Kong", pues es, en gran medida, un producto de un entorno y de una política social particular - una pequeña "ciudad-Estado" con una cultura característica y una maquinaria administrativa altamente eficiente, que opera en una sociedad caracterizada por un elevado crecimiento económico sostenido. La ICAC contaba con buenos recursos y empleaba personal de apoyo y expatriado. Tenía programas de selección y formación intensiva y sus programas de educación pública eran excelentes. Mientras que era, por tanto, un modelo relativamente caro de emular, vale la pena señalar el comentario de DE SPEVILLE de que la ICAC no era la estrategia en sí misma, sino que era un mecanismo para implementar una estrategia más amplia que incluía la aplicación del Derecho y conseguir el apoyo popular.

\section{Mali y Senegal: determinantes del fraude aduanero}

David Stasavage y Cecile Daubree (Stasavage y Daubree, 1997) examinaron los determinantes del fraude aduanero en los Estados africanos occidentales de Mali y Senegal, que tienen muchas similitudes en cuanto a herencia colonial, estructura estatal y estilo político, son miembros de la zona Franca y tienen políticas proteccionistas. El fraude aduanero es un ejemplo tanto de la pérdida de ingresos, como de corrupción. El fraude puede ser de varios tipos e incluye la declaración de mercancías por debajo de su valor real, clasificación errónea y pago de impuestos por debajo de lo debido. Pero sea cual sea la forma en la que tiene lugar, puede tener consecuencias económicas significativas para tales Estados en vías de desarrollo, puesto que la base de ingresos del Estado depende en gran medida de la imposición eficaz sobre el comercio.

STASAVAGE y DAUBREE emplearon modelos estándar centrados en un agente principal como base de su investigación, aunque se aceptó que tales modelos se centraran en factores de nivel nacional y que no pueden predecir los resultados para administraciones de aduanas concretas. En su lugar, trataron de comparar el fraude producto por producto dentro de las administraciones de aduanas. Sus conclusiones generales fueron que la liberalización del comercio había reducido los niveles de fraude y que la inspección previa del cargamento "puede ser una herramienta poderosa para reducir el fraude». Además, para reducir el fraude en las aduanas, los benefactores deberían apoyar reformas institucionales que reduzcan la discrecionalidad de los empleados públicos y mejoren el seguimiento. El fraude aduanero es en sí mismo un síntoma de los fracasos políticos e institucionales nacionales concretos. STASAVAGE sugirió que la magnitud de los salarios de los empleados públicos no era tanto un tema importante en Mali y Senegal, si se comparaba con otros países africanos. En su lugar, un problema clave con los servicios de aduanas en Mali y Senegal era la falta de fondos para gastos corrientes no salariales, tales como necesidades básicas (incluidos el combustible, vehículos y otros costes administrativos) para que los empleados hicieran su trabajo. Además, tanto en Mali como en Senegal, los cambios en las políticas comerciales estaban relacionados con la caída y auge de los diferentes grupos empresariales. Algunos grupos de industriales y comerciantes estaban perdiendo su fuerza política para demandar protección. La liberalización estaba creando nuevos grupos empresariales en los Estados, que estaban influenciando las políticas públicas.

En relación con una posible importante reforma -el empleo de compañías de inspección - STASAVAGE sostuvo que había una tensión entre los costes de tales servicios de inspección previa y los ingresos que se obtenían de esta actividad. Las mejoras conseguidas con los servicios de inspección previa se basaban en parte en el acompañamiento de reformas en los propios servicios aduaneros, concretamente mejoras en la recogida y valoración de información y en la informatización de los servicios.

\section{Tanzania}

El Embajador Alexander Muganda (Muganda, 1997) describió las circunstancias que rodeaban el establecimiento y los resultados del informe Warioba sobre la corrupción en Tanzania, que fue terminado en enero de 1996 y se presentó en diciembre de 1996. J. S. Warioba, anterior Primer Ministro y primer Vicepresidente, había presidido la Comisión Presidencial; Muganda había sido tanto el Secretario de la Comisión, como el Director de la Oficina de Prevención de la Corrupción en Tanzania. 1994 fue un año clave en Tanzania debido a los escándalos de corrupción y las elecciones de 1995 dieron lugar a compromisos anticorrupción. La Oficina de Prevención de la Corrupción era ineficaz, pero un problema adicional era la falta de voluntad política para otorgar a la Oficina fuerza para actuar. La Oficina necesitaba una reorganización completa y más poder. Una cuestión importante respecto de la corrupción en Tanzania eran los vínculos clientelistas entre los hombres de negocios y los que tomaban las decisiones políticas, concretamente los políticos. Estos vínculos habían hecho aumentar en gran medida la corrupción en los últimos años.

El informe Warioba había identificado "una corrupción galopante en el funcionariado" que se había intensificado a un ritmo alarmante en las pasadas dos décadas. Había tanto una corrup- 
ción a «pequeña» como a «gran» escala en los niveles públicos más altos. La corrupción en pequeña escala estaba ampliamente extendida. M uganda dijo que era «una incomodidad seria» y que había «subvertido la prestación de servicios efectiva». Se descubrió en la policía y en los servicios judiciales y en todos los sectores sociales. De las pruebas obtenidas en las audiencias públicas, la corrupción en pequeña escala afectaba a la mayoría delos miembros del funcionariado y era la principal fuente de descontento público. La corrupción a gran escala se había identificado en la obtención de bienes y servicios, en la distribución de licencias para cazar y extraer minerales, y en los contratos públicos importantes, en particular en la construcción de carreteras y en las obras públicas. Una evaluación de 24 contratos de construcción descubrió unos excesos sustanciales en los costes; los costes se habían incrementado de 97,4 millones a 154,7 millones de dólares americanos. Aquí se podía apreciar una «nferencia de la corrupción», dijo Muganda.

Se identificaron algunas de las causas del crecimiento de la corrupción, incluido el deterioro económico, el declinar de la moral pública y la carencia de un liderazgo político en este tema. La Comisión Warioba había hecho al gunas recomendaciones para mejorar la integridad pública, que incluía la propuesta de enmiendas a la Ley del del Código Etico de la Clase Política, el veto a corto plazo de los empleados públicos, una acción disciplinaria contra la policía y la judicatura y la revitalización de la 0 ficina de Prevención de la Corrupción. Respecto de cuestiones a largo plazo, también merece la pena comentar el sistema jurídico de Tanzania. Algunas leyes crearon oportunidades que favorecían la corrupción; debían ser enmendadas. Una últimaárea deimportancia era la «ngeniería social». Se necesitaban medidas de este estilo: educación pública, anuncios sobre integridad pública positiva y anticorrupción, así como medidas generales para incrementar la conciencia pública. En el caso de Tanzania, era importante recordar o educar a los ciudadanos para que se quejaran de las actuaciones corruptas e intentar evitar que los políticos acabasen adquiriendo los malos hábitos de la corrupción. concretas de un país. Además, se debe prestar mucha más atención a las cuestiones de duración, secuencia y coherencia en el planteamiento, los detalles de la reforma y su sostenibilidad y la voluntad política y de gestión excepcional necesaria para promover y apoyar la reforma en esta área. En M ali y Senegal, la liberalización económica parece haber ayudado a reducir el fraude aduanero, cuando se combina con la reforma institucional y los salarios relativamente altos. En Tanzania, la liberalización económica es parte de la explicación del crecimiento de la corrupción en pequeña y gran escala. Algunos Estados se han embarcado en programas de reforma globales: en Ecuador y Tanzania, las estrategias anticorrupción incluyen expectativas de los efectos ben eficiosos de los esfuerzos de crecimiento y alfabetización a largo plazo, combinados con reformas legales y el fortalecimiento institucional. Pero estos catálogos plantean importantes cuestiones sobre las prioridades a corto plazo y las interrelaciones entre tales esfuerzos de reforma. Casi todas las experiencias analizadas en el grupo de trabajo de París plantearon de una forma u otra el tema de la voluntad política y de gestión: ituvo la clase política un compromiso sostenido con la reforma anticorrupción o se comprometió en un ejercicio meramente «cosmé tico»? En Hong Kong, el compromiso político excepcionalmente alto, el apoyo popular, la buena organización y una estrategia coherente han tenido buenos resultados, incluso hay cuestiones relativas a la sostenibilidad y posibilidad de transferir tal planteamiento. El caso de Botswana, con un vehículo equivalente para trabajar en el ámbito anticorrupción, gestionado por algunos empleados de ultramar, sugiere que puede haber datos de que sea aplicable en cualquier parte, pero su éxito se deberá medir, no sólo por la eficacia del sistema judicial, sino también por el continuo apoyo político; así se investigan figuras senior de la vida política y pública. El reciente esfuerzo anticorrupción de Tanzania, con su proceso de investigación, debe convertirse en un compromiso político e institucional sostenido. El caso de Ecuador claramente ilustra los resultados del desorden político que puede causar la corrupción de gran calado.

\section{Discusión de los casos estudiados}

Del estudio de las experiencias de estos países concretos, es posible sostener que es difícil confiar excesivamente en una moda concreta sobre las estrategias anticorrupción, una idea innovadora particular o un modelo de análisis de la corrupción. Una estrategia anticorrupción se debe diseñar comprendiendo que las causas específicas, las ocasiones y las medidas correctivas adecuadas están directamente relacionadas con las circunstancias

\section{Diversidad y búsqueda de la integridad pública}

El tema de la diversidad de la corrupción es importante. Hay distintas causas y consecuencias de la corrupción. Los diferentes resultados dela corrupción y el consecuente escándalo tienen trascendencia en términos tanto de los costes a corto plazo, como de los presuntos ben eficios de desarrollo a largo plazo. En al gunas sociedades, como en Asia oriental, la corrupción puede estar generalizada (aunque «aislada» dentro de una esfera política distinta), 
pero no parece ser una barrera al crecimiento económico rápido, mientras que la corrupción es una parte integral del estancamiento económico y la decadencia del África Subsahariana y ha tenido efectos dañinos en Latinoamérica (DIAMOND, 1987; KPUNDEH, 1995; LitTLE Y POSADA-Carbo, 1996; Banco Mundial, 1994; Banco Mundial, 1997; SABA y MANZETTI, 1996). Igualmente, los cambios en la políitica a nivel estatal, subestatal y micro, han creado en décadas anteriores y continúan creando nuevos incentivos y oportunidades en los niveles, modelos y predominio de la corrupción. Por tanto, la upreocupación inmediata del gobierno chino por la economía en fase de estancamiento y, en consecuencia, por la necesidad de revitalizar la sociedad" (puesto que ha "relajado su control sobre la distribución de los recursos, ha dado gran autonomía a las autoridades locales para invertir directamente y ha descentralizado su poder de gestión sobre las empresas» en el sector económico) no condujo sólo a la reactivación de los vínculos de antiguos modelos de cliente, sino también de los nuevos patrones de corrupción (GONG, 1993, pp. 317, 323-4).

Además, la diversidad de la corrupción -y las perspectivas y planteamientos de aquellos que la reportan o analizan - ha tenido un impacto sobre los intentos de reducir o minimizar los efectos de la corrupción a través de las estrategias anticorrupción.Con frecuencia, la estrategia propuesta se basa en un punto de vista diferente que tiene que ver con las causas y naturaleza de la corrupción. Ha habido probablemente tres planteamientos distintos ante la corrupción contemporánea y las estrategias anticorrupción; los análisis económicos; la opinión pública o cultura cívica; y los puntos de vista institucionales. Los análisis económicos priorizan la relación del "agente-principal" para identificar la corrupción y las estrategias anticorrupción, mientras que las perspectivas de la opinión pública o de la cultura cívica examinan el contexto social o cultural de la corrupción y sugieren, como resultado, un cambio accitudinal en las masas o una toma de conciencia civil en las estrategias anticorrupción. El punto de vista institucional presta atención tanto al fortalecimiento de las instituciones (auditor general o funciones de los organismos anticorrupción), como a los controles y procedimientos dentro de las instituciones, como los códigos y registros de interés, para definir las expectativas de los políticos y de los funcionarios públicos. Por tanto, la estrategia de KLITGAARD se basa en el análisis económico del agente principal y se ha evaluado en algunos contextos de paises diferentes en vías de desarrollo (KLITGAARD, 1988). De forma amplia, ha habido también cuatro niveles en la estrategia anticorrupción: internacional, nacional, local y populista (THEOBALD, 1990). En el período que va desde mediados de los noventa, han surgido una serie de iniciativas anticorrupción internacionales (KAUfMANN, 1997; RILEY, 1998, ROSE-ACKERMAN, 1997). Las décadas anteriores han visto estrategias basadas en la acción nacional y local, incluidos los organismos anticorrupción, investigaciones públicas, procedimientos de queja y campañas de concienciación pública (CLARKE, 1983; HeIDENHEIMER et al.,
1989). Una condición necesaria pero no suficiente para reducir la corrupción sería el desarrollo de una sociedad civil vigorosa, que abarque una pluralidad de grupos organizados independientemente, capaces de satisfacer sus necesidades e intereses. Las iniciativas populistas tales como las purgas de los funcionarios públicos no han tenido mucho éxito, aunque el tema de la corrupción ha adquirido una gran relevancia política en los últimos años debido a las acciones de las ONGs y otros grupos de interés público (Harsh, 1993; Guhan y Paul, 1997; Riley, 1998; TheOBALD, 1990).

Una complicación adicional ha sido el crecimiento de nuevas preocupaciones 0 actividades que tienen un impacto cada vez mayor sobre los tipos y niveles de la corrupción. Se presta una atención cada vez mayor a las actividades de las multinacionales y otros negocios de exportación que han explotado tradicionalmente las insuficiencias legales del propio país, diferentes expectativas culturales y procesales, y la aprobación políica tácita de la ventaja comercial mientras que los propios gobiernos han empleado métodos similares a la hora de poner en práctica los objetivos directos de asuntos exteriores. En el caso de la corrupción en las rutas de paso en los Estados caribeños y bálticos, la existencia de un sector público débil o corrupto y, por tanto, la presencia de un proceso de toma de decisiones paralelo que favorece a ciertas secciones de la sociedad, puede permitir que los criminales: continúen en el pás y que prosiga la actividad criminal internacional creando «refugios para delitos»; aceleren tanto el crimen general y la corruptibilidad judicial; afecten de forma adversa los ingresos del Estado mediante los vínculos entre la corrupción, la diferenciación impositiva entre fronteras y las rutas de contrabando; y subviertan la regulación de servicios financieros débiles, al crear oportunidades para el fraude en la inversión, lavado de dinero y movimientos de capital ilícito.

Puede sostenerse que es difícil tanto determinar las causas 0 consecuencias de la diversidad de la corrupción, como tratar de diseñar las estrategias comunes para abordarla. Valorar la corrupción en términos de instrumentalidad -incentivo, oportunidad y riesgo - o en términos de escenario - categorización o caracterización de los actores - también corre el riesgo de tratar de imponer una construcción analítica artificialmente uniforme. Igualmente, las perspectivas históricas, de definición o culturales juegan un papel, pero sólo en términos de perspectivas a largo plazo sobre los cambios en las normas culturales y de la sociedad. Las cuestiones inmediatas deben ser qué tipo o tipos de corrupción son los más perjudiciales; qué relaciones hay entre la corrupción y un gobierno sólido y los objetivos de un buen sistema de gobierno; cómo puede actuar un Estado capaz y eficaz para reducir la corrupción; y qué formas de estrategia anticorrupción son más probables que sean las más eficaces a corto y largo plazo para minimizar la corrupción, concretamente para aquellos más adversamente afectados por sus consecuencias, directa o indirectamente. 
D entro de tal contexto, la importancia de captar la diversidad internacional de la corrupción llega a estar clara cuando se analizan las mejores y las peores prácticas. Es importante reconocer que si los Estados se toman en serio la lucha contra la corrupción requerirán una valoración detallada de los costes de la corrupción en cada país. Esto debe incluir una valoración de dónde es más probable que la corrupción imponga los mayores costes, incluidos los ingresos fiscales y aduaneros, la regulación de los negocios, el patrocinio del Estado de los proyectos de infraestructuras, reforma institucional, el compromiso político y la implicación pública (RoSE-AckERM AN, 1997). I gualmente, el planteamiento frente a las estrategias anticorrupción requiere planificación, una valoración meticulosa, una estrategia que se centre en los sistemas corruptos y no sólo en los individuos corruptos y una implementación eficaz (KLITGAARD, 1997).

Para la mayoría de países en vías de desarrollo, el objetivo a corto plazo debería ser encontrar los medios para establecer las medidas económicas más eficaces para controlar la corrupción. Cómo se llega a esto depende de algunos factores, incluido el legado de anteriores campañas anticorrupción, la reacción de la población a las investigaciones anticorrupción y la imparcialidad de tales investigaciones (FIn L LAY y SteWART, 1992).

\section{La elección de la estrategia}

Por tanto, hay algunas cuestiones marco que deberían abordarse al planificar estrategias anticorrupción a corto plazo para identificar medidas sostenibles y rentables que tengan impacto. Éstas incluyen las siguientes.

\section{Cargo o titular del cargo}

¿H abría que poner el énfasis en el cargo o en su titular? La formación de los funcionarios les dota de habilidades transferibles que se valoran en cualquier parte del sector público y también en el sector privado, concretamente en relación con la imposición fiscal, el derecho y la auditoría. Al mismo tiempo, los funcionarios formados que trabajan dentro de una organización inflexible o incapaz de conseguir resultados llegan a desmotivarse. Por otra parte, formar a las organizaciones 0 a los empleados públicos en los procedimientos puede ayudar a la sostenibilidad de las competencias, concretamente si a los nuevos empleados se les forma en prácticas organizacionales que son menos trasladables, pero que pueden inhibir la iniciativa de los funcionarios 0 implicar una falta de fe en su capacidad para llevar a cabo sus obligaciones sin tal entorno de control. También debería haber «esposas de oro» - alicientes atractivos para retener al personal ya formado- o se debería poner más énfasis en su desempeño, imponiendo sanciones ahí donde el criterio principal es el no actuar según los requerimientos de la organización- y hasta dónde se debería formar a las organizaciones en la evaluación de las competencias y en la evaluación de su personal.

\section{Ingreso 0 gasto}

El tema aquí es si toda estrategia anticorrupción se debería centrar en proteger los ingresos del Estado - en la integridad y competencia de los organismos fiscales y aduaneros y los roles de los Auditores G enerales- o en investigar las pruebas de actividad criminal, como la corrupción pactada. Lo primero puede ayudar a desarrollar una base de ingresos estable, mientras que lo segundo puede demostrar más visiblemente el compromiso del Estado para usar de forma eficaz los recursos y de abordar la corrupción (investigar y perseguir judicialmente la corrupción ge neralmente significa que el dinero se ha gastado o invertido y a menudo se confía en la velocidad e integridad del sistema de justicia criminal para su implementación).

\section{Restitución o retribución}

¿C uál es el propósito de la búsqueda de la integridad pública? ¿Es la retribución - el castigo visible de los corruptos- o la restitución (proteger los fondos del Estado y tratar de devolver al Estado aquellos fondos y pagos corruptos relacionados con ellos adquiridos ilegalmente? M ientras que lo primero supone confiar en un sistema de justicia penal interno eficaz, lo segundo no requeriría sólo de sistemas eficaces en otros países, sino de organismos encargados de seguir la pista de los bienes, embargos y confiscaciones.

\section{Vehículo de reforma}

Algunosreformadoresson partidarios del establecimiento deun organismo anticorrupción; otros sugieren desarrollar un planteamiento basado en departamentoso funciones más vulnerables. En al gunosEstados, sehaevaluado el riesgo delosdepartamentos, procedimientos y actividades que son más vulnerables ante la corrupción con el objetivo de identificar dónde puede tener un impacto más corrosivo la corrupción. Ésteha sido el resultado final de destinar recursosy adoptar medidas. Laalternativa es establecer un organismo anticorrupción con un propósito gl obal paraacometer investigaciones en cualquier parte dentro del sector público. Sin la debidaplanificación delosnegocios, por ejemplo, estosorganismos llegar a ser reactivoso sometersealainfluencia políticay, careciendo demedios para evaluar la gestión, centrarse en áreas erróneas, con 
propósitos erróneos y con resultados erróneos. Existen, por supuesto, algunas alternativas privadas o no estatales, incluida la gran participación de las firmas de inspección, de gestión de los expatriados en las agencias del gobierno u ONGs y otros organismos externos en proyectos, por ejemplo, Build-Operate-Transfer (BOT). También puede haber sitio para proyectos que se centren menos en el riesgo que en la oportunidad de tratar de combatir la corrupción mediante la prestación de información accesible de los servicios de aduanas, el benchmarking (o criterios de comparación) y la publicidad. De igual manera, el incremento de la corrupción y el fraude para financiar la actividad de los partidos políticos podría verse constreñido por la financiación estatal de los partidos, la publicación de listas de nombramientos y controles sobre el contacto político con los departamentos del gobierno.

\section{Midiendo el éxito}

A menudo un problema clave es cómo medir el éxito, lo cual puede ser excepcionalmente difícil. Cómo se debe medir la corrupción y su prevención, la detección, investigación y procesamiento judicial es el tema de muchos debates académicos y profesionales. Un aspecto emergente de este debate tiene que ver con la cuestión de si se debería poner la atención en la corrupción de la calle o en la corrupción a gran escala. Diseñar un sistema para luchar contra la corrupción se considera efectivo, pero es difícil medir sus resultados. Una relación más responsable y abierta entre el ciudadano y el Estado a menudo es clave en esta cuestión. Construir sistemas de integridad nacional es un objetivo más a largo plazo que puede generar expectativas a corto plazo, pero que de no cumplirse conducen a la frustración de los ciudadanos. Enfrentarse al blanqueo de dinero, al fraude fiscal y al crimen organizado en algunos países en vías de desarrollo puede ser parte de una respuesta internacional, pero es una cuestión de en qué medida esa respuesta está dictada por los requisitos de los países que dan financiación y dónde se obriene el mayor beneficio.

\section{Coordinación de los benefactores}

Uno de los temas más interesantes del grupo de trabajo de París UNDP/OCDE era el de los esfuerzos de los benefactores en este campo. Virtualmente, todos los benefactores tienen actualmente políticas sobre corrupción, algunas de las cuales existen desde hace mucho tiempo y otras que se han desarrollado a mitad de los años 90 . Hay una serie de preguntas importantes acerca de cómo se deben coordinar los esfuerzos de los benefactores. ¿Quién determina la cooperación y las responsabilidades entre organismos y, por tanto, la titularidad de la responsabilidad y de los medios para luchar contra la corrupción?

Tales temas marco son cruciales para desarrollar una estrategia sistemática a largo plazo. Mientras que el modelo ICAC de
Hong Kong puede ser demasiado caro y demasiado amenazador políicamente para muchos países, y otros ejemplos de organismos independientes demasiado limitados o demasiado constreñidos por un control ministerial, un organismo anticorrupción cuya agenda y atención viene determinada desde el principio tras una consulta interna y externa, puede ofrecer la oportunidad con el apoyo del benefactor de brindar un bloque de construcción genérico e inmediato para el buen gobierno allí donde otras reformas administrativas serían problemáticas. El planteamiento global de la reforma del sector público que abarca la elaboración de un nuevo borrador y puesta al día de la legislación y la infraestructura judicial, además de la formación, organización y responsabilidad de un reducido número de departamentos del sector público, también es costoso, lleva tiempo y es susceptible de sufrir un retraso sustancial. Por otra parte, el planteamiento del enclave de transferir actividades a unos organismos semiautónomos dirigidos y financiados externamente es, a menos que el apoyo gubernamental futuro a largo plazo sea cierto, una solución que puede generar interferencias políticas a corto plazo, resentimiento entre otros departamentos y la posibilidad de perder personal experimentado a favor de otras organizaciones (DEMONGEOT, 1994; DiA, 1993).

Las iniciarivas anticorrupción más recientes buscan un planteamiento más integrado, preferiblemente centrado en identificar estrategias eficaces y a corto plazo que distingan una acción complementaria que se deba acometer a varios niveles: al nivel de los ciudadanos o popular, institucional o procedimental y al nivel de los funcionarios senior, y de los contratos más sustanciosos. Sin embargo, tales iniciativas deben determinar primero cómo equilibrar el trabajo mediante las instituciones existentes con grupos o movimientos de participación; segundo, tales iniciativas deben ser conscientes de que los esfuerzos del gobierno en promover el desarrollo económico o la reforma administrativa estructural pueden en sí mismos introducir nuevos actores o modelos de corrupción a medida que el desarrollo hace disminuir o deja obsoleta la corrupción existente; tercero, deben tener en cuenta las actitudes tradicionales, culturales y sociales para asegurar que las reformas se consideran deseables, aceptables y practicables.

Por tanto, al buscar objecivos de desarrollo político y económico, tanto voluntariamente como bajo la presión ejercida por organismos externos, los gobiernos deben valorar los medios más apropiados para iniciar, implementar y sostener la reforma, además de poner la atención en la coordinación y seguimiento del cambio.

Por tanto, se debe dar la debida importancia al grado en el que se pueden introducir reformas procedimientales o estructurales para limitar las oportunidades e incentivos que favorezcan la corrupción y generar el riesgo de detección, al tiempo que se hace que los ciudadanos se impliquen, planteen sus quejas y que les sean respondidas satisfactoriamente y se evalúan los medios apropiados en términos de coste, impacto, eficacia y sostenibili- 
dad, además de las relativas fortalezas políticas y financieras de aquellos involucrados en la corrupción. Con frecuencia, los organismos anticorrupción pueden llevar a cabo eficazmente estas tareas y podrían ser importantes para apuntalar y mantener la atencion cada vez mayor que se le ha dado a otras respuestas genéricas - las iniciativas a nivel del ciudadano, como parte de los pasos dados hacia el desarrollo de la participación, investir de autoridad a aquellos más adversamente afectados por la corrupción estatal y los más alienados por el propósito de reforma-. Estas medidas pueden incluir: disposiciones legislativas; procedimientos administrativos simplificados y controles de vigilancia; información accesible, el sistema de aduanas y su aplicación, el benchmarking (o criterios de comparación y estudios de prestación de servicios); quejas y respuestas rápidas y eficaces (líneas directas, descubrimiento desituacionesilegales, D efensor del pue blo y tribunales administrativos); implicación de la comunidad (concienciación y educación, transparencia de acceso a la información, grupos de vigilancia de los ciudadanos); y asegurar la independencia de los medios. Muchas de estas reformas institucionales se asocian con las ideas de la «N ueva Gestión Pública» en las sociedades occidentales y como tales, están sometidas a varias críticas (H ILL, 1997).

Las iniciativas a nivel de ciudadano también tienen tres roles adicionales en términos del sistema de gobierno. Primero, tienen la finalidad de centrarse en los miembros más pobres de la sociedad, controlando o minimizando la corrupción a pequeña escala mediante la protección contra la naturaleza arbitraria del Estado y desusfuncionarios, concretamenteen relaciones entre la sociedad y el Estado, incluida la reforma estructural (los efectos deladescentralización y la desregulación). Segundo, fomentan las mejoras en la actividad administrativa básica, centrándose en el ciudadano como consumidor y cliente mientras que ofrece una medición de la gestión fácil de valorar y ponea disposición del dominio público la información, el control sobre el proceso y el derecho de que sus quejas sean atendidas; la ausencia anterior de estos elementos ha motivado la existencia y persistencia de la corrupción de nivel inferior. Tercero, implican a la sociedad de forma más directa que la conseguida mediante la participación a través de los partidos políticos, a un nivel más local y con un beneficio inmediato para ellos mismos y sus comunidades.

Se han desarrollado proyectos más integrados que abarcan algunas iniciativas, incluidas las campañas anticorrupción y los roles de los organismos en el contexto de lo que se denomina Sistemas de Integridad N acional, donde se sostiene que los «íderes en la campaña contra la corrupción sólo pueden alcanzar beneficios reales cuando la sociedad cambia colectivamente sus expectativas de comportamiento público, o cuando la gente cree que tiene derecho a esperar que un gobierno no sea corrupto» y que trabaja «en colaboración con el gobierno y la sociedad civil» mediante la concienciación de la sociedad civil, la construcción de instituciones, la prevención de las prácticas corruptas y el enjuiciamiento de los funcionarios corruptos y donde las voluntades políticas y públicas se sostienen por una integración organizacional y proce dimental adecuada (LanGSETH y otros, 1997).

\section{Conclusión}

Si hay diversidad tanto en las antiguas como en las nuevas formas de corrupción, se deberían tener en cuenta a la hora de estudiar las estrategias anticorrupción. Los casos analizados en el Grupo de Trabajo de PD N U/OCDE ilustran tanto los antiguos orígenes, como la vitalidad de las nuevas formas de corrupción y la búsqueda de la integridad pública. Sin embargo, todos los casos muestran un planteamiento esencial de cualquier estrategia para que tenga éxito: se precisa de una voluntad política y de gestión excepcional para promover y mantener la reforma anticorrupción, incluso aunque los casos estudiados ponen de manifiesto las dificultades de las soluciones universalistas. No obstante, si se precisan otros elementos comunes de toda estrategia universal, entonces se pueden hacer algunas sugerencias básicas. Además de la mejora institucional, en todas las sociedades, las profesiones deberían fortalecerse - aumentando su excelencia, independencia y habilidades técnicas- como parte de una estrategia para controlar la corrupción. Los abogados, contables, pe riodistas de investigación, todos necesitan ayuda. Es más probable que en las sociedades democráticas se materialicen las habilidades profesionales, además de la voluntad política y de gestión para controlar la corrupción, allí donde las presiones de la competición política fuerzan a menudo a los políticos a actuar. Por tanto, la democratización es una condición necesaria, pero no suficiente, para reducir la corrupción. La liberalización económica no es una simple panacea para la corrupción en el sector público. Pero reducir el tamaño del Estado también conlleva disminuir la magnitud de los potenciales «ingresos» corruptos y permite al sector público acercarse al ideal contemporáneo del estado eficiente y con capacidades. La «erupción de la corrupción» - la conciencia actual de la corrupción debido a los escándalos a los que se les ha dado mucha publicidad- asegurará que se están desarrollando medios más eficaces de reducir la corrupción. 


\section{Bibliografia}

ANDRESK, A. Parasitism and Subversion. London: Weidenfeld and Nicolson, 1970.

AskIN, S. y C. CourNs. «External Collusion with Kleptocracy: Can Zaire Recaprure its Stolen Wealth?, Review of African Political Economy, no. 57, 1993.

Charlicx, R. «Corruption in Political Transition: a Governance Perspectiven. Corruption and Reform, Vol. 7, No. 3, 1993.

Clarke, M. (ed.) Corruption Causes, Consequences and Control, London: Frances Pinter, 1983.

Demongeor, P. «Market-oriented approaches to capacity building in African. Public Administration. Vol. 14, no. 5, 1994.

DiA, M. "A Governance Approach to Civil Service Reform in Sub-Saharan Africa". Documento Técrico del Banco Mundial Número 225. Washington, D.C.: Banco Mundial. 1993.

Diamond, L. "Class Formation in the Swollen African State". Joumal of Modern African Studies, Vol. 25, no. 4, 1987.

DoIG. R.A. "Good Government and Sustainable Anti-Corruprion Strategies: a Role for Independent Anti-Corruption Agencies?". Public Administration and Development. Vol 15, No. 2, 1995.

Findlay, M. and StewarT, A. "Implementing Corruption Prevention Strategies Through Codes of Conduct." Corruption and Reform Vol. 7, No. 1, 1992.

FRIMPONG, K. "An Analysis of Corruption in Botswana." Grupo de Trabajo del Centro de Desarrollo de UNDP-PACT\&OCDE sobre Corrupción e Iniciativas de Mejora de la Integridad en el contexto de Economías en Desarrollo. París, 1997.

GonG, T. "Corruption and Reform in China: An Analysis of Unintended Consequences." Crime, Law and Social Change. Vol. 19, No. 4, 1993.

Goudie, A. and StasavaGe. "Corruption: the Issues", Documento Técnico del Centro de Desarrollo de la OCDE, no. 122, 1997.

Guhan, S. and Paul, S. (eds.) Corruption in India: An Agenda for Action, New Delhi: Vision Books, 1997.

HampTon, M. The Offshore Interface: Tax Havens in the Global Economy, Basingstoke: Macmillan, 1996.

HaYward, P. (ed.) "Political Corruption," número especial de Political Studies, Vol. 45, no. 3, 1997.

HarSCH, E. "Accumulators and Democrats: Challenging State Corruption in Africa." Journal of Modern African Studies, Vol. 31, No. 1, 1993.

Heidenheimer, A. J. et al. Political Corruption: A Handbook, New Jersey: Transaction, 1989.

HILL, M. The Policy Process in the Modern State, London: Prentice Hall, 1997.

HOPE, K. R. "Administrative Corruption and Administrative Reform in Developing Countries." Corruption and Reform. Vol. 2, No. 2, 1987.

Huntington, S. Political Order in Changing Societies. Yale: Yale University Press, 1968.

IDS. "Liberalisation and the New Corruption." IDS Bulletin. Vol. 27, no. 2, 1996.

IRIS. Governance and the Economy in Africa: Tools for Analysis and Reform of Corruption, Center for Institutional Reform and the Informal Sector [IRIS], University of Maryland, 1996.

Kaufmann, D. "Corruption: The Facts". Foreign Policy, 107, 1997.

KLITGaARd, R. Controlling Corruption, Berkeley: University of California Press, 1988.
KuTGaARD, R. Cleaning UP and Invigorating the Civil Service. Washington: Banco Mundial, 1997.

Kpunder, S. J. Politics and Comption in Africa: A Case Study of Sierra Leone, Lanham: Universiry Press of America, 1995.

LANCSETH, P and Rick Stapenhurst. The Role of the National Integrity System in Fighting Corruption EDI Staff Working Paper, Washington, D.C.: World Bank, 1997.

LARREA-SANTOS, R. "Cotruption and Integrity Iniciatives Implemented in Developing Countries." Grupo de Trabajo del Centro de Desartollo de UNDP. PACT\&OCDE sobre Corrupción e Iniciativas de Mejora de la Integridad en el contexto de Economías en Desarrollo. París, 1997.

LEV, M. Regulating Fraud. London: Tavistock, 1987.

LITTLE, W. and Posada-Carbo, E. (eds.) Political Corruption in Europe and Latin America, London: Macmillan/lLAS, 1996.

Muganda, A. A. "The War Against Corruption in Tanzaia: Overview of the report of the Presidential Commission on Corruptionn. Grupo de Trabajo del Centro de Desarrollo de UNDP-PACT\&OCDE sobre Corrupción e Iniciativas de Mejora de la Integridad en el contexto de Economías en Desarrollo. París, 1997.

NoOnan, J. T. Jr. Bribes. New York: Macmillan, 1984.

OECD. The Final Report of the Ad Hoc Working Group on Participatory Development and Good Governance of the Development Assistance Committee. OCDE. París, 1997.

ReEd, Q. "Transition, Dysfunctionality and Change in the Czech and Slovak Republics". Crime, Law and Social Change. Vol. 22, No. 4, 1994.

RlLeY, S.P. [1983]. "The Land of Waving Palms': Corruption Inquiries, Political Economy and Politics in Sierra Leone," in M. Clarke, (ed.) Corruption: Causes, Consequences and Control, London: Frances Pinter, 1983.

RuLEY, S. "Political Adjustment? Democratic Politics and Political Choice in Africa". Centre of African Studies Seminar Paper. University of Liverpool: Liverpool, 1992.

RILEY, S.P. «Post-Independence Anti-corruption Strategies and the Contemporay Effects of Democratisation." Corruption and Reform, Vol, 7, no. 3, 1993.

RILEY, S.P. "The Politicial Economy of Anti-corruption Strategies in Africa." European Journal of Development Research, Vol. 10, no. 1, 1998.

Rose-ACKerman, S. Comruption: A Study in Political Economy. New York: Academic Press, 1978.

Rose-Ackerman, S. Corruption and Development. Anual Bank Conference on Development Economics. Washington: Banco Mundial, 1997.

SABA, R. and MANZETT, L. "Privatisation in Argentina". Corruption and Reform. Vol. 25, no. 4, 1996.

SANDBROOK, R. The Politics of Africa's Economic Stagnation. Cambridge: Cambridge University Press, 1985.

SEN, A. K. "On Corruption and Organised Crime", en el Programa de Control de Drogas de las Naciones Unidas [UND.C.P], World Drug Report, Oxford: Oxford University Press, 1997.

Serageldin, I. and Landell-Mills, P. "Governance and the External Factor". Conferencia Anual de Economía y Desarrollo. Banco Mundial: Washington, D.C., 1991.

ShLEIFER, A. and VISHNY, R. "Corruption," Quarterly Journal of Economics. Vol. 108, no. 3, 1993. 
SPEVILLE, B. de. «H ong Kong's War on Corruption». Grupo de Trabajo del Centro de D esarrollo de UN D P-PACT \& O CDE sobre Corrupción e Iniciativas de M ejora de la Integridad en el contexto de Economías en D esarrollo. París, 1997.

Stasavage, D. and Daubree, C. «T he D eterminants of Customs Fraud: Evidence from Senegal and Mali». UN D P.PACT \& OECD D evelopment Centre Workshop on Corruption and Integrity Improvement Iniciatives in the C ontext of D eveloping Economies. París, 1997.

TheOBALD, R. Corruption, Development and Underdevelopment. Basingstoke: Macmillan, 1990.

Theo BaLD, R. «Corruption in a Changing World: Comparisons, Theories and Controlling Strategies». The $3^{\text {rd }}$ International Conference on Ethics in the Public

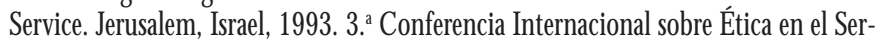
vicio Público.

Trang, D. Corruption and Democracy. Institutional and Legislative Policy, Hungría, 1994.

UN D P. Human Development Report. O xford: O xford University Press, 1997.
D epartamento de Cooperación Técnica de las $N$ aciones U nidas y Centro de D esarrollo Social y Asuntos H umanitarios para el D esarrollo. Report of an Interregional Seminar, 1989.

United Nations International D rug Control Programme. Informe M undial sobre D rogas. 0 xford: O xford University Press, 1997.

W ADE, R. «T he M arket For Public O ffice: W hy India is N ot Better at D evelopment». World Development, Vol. 13, N 0. 4, 1985.

Werner, S. B. «The Development of Political Corruption: A C ase Study of I srael.» Political Studies. Vol. 31, no. 4, 1983.

W ORLD BAnk. M anaging Development: The Governance Dimension. World Bank: Washington, D.C., 1991.

-----. Adjustment in Africa. Oxford: 0 xford University Press, 1994.

World D evelopment Report: The State in a Changing World. 0 xford: 0 xford University Press, 1997. 